\title{
Correlations and entanglement in probability theory
}

\author{
E. G. Beltrametti ${ }^{\dagger}$ and S. Bugajski ${ }^{\ddagger *}$ \\ ${ }^{\dagger}$ Department of Physics, University of Genoa, and \\ INFN-Sezione di Genova \\ ${ }^{\ddagger}$ Institute of Physics, University of Silesia, and \\ Institute of Pure and Applied Computer Science, \\ Polish Academy of Sciences
}

\begin{abstract}
We generalize the classical probability frame by adopting a wider family of random variables that includes nondeterministic ones. The frame that emerges is known to host a "classical" extension of quantum mechanics. We discuss the notion of probabilistic correlation and show that it includes two kinds of correlation: a classical one, which occurs for both deterministic and indeterministic observables, and a nonclassical one, which occurs only for indeterministic observables. The latter will be called probabilistic entanglement and represents a property of intrinsically random systems, not necessarily quantum. It appears possible to separate the two kinds of correlation and characterize them by numerical functions which satisfy a simple product rule.
\end{abstract}

\section{Introduction}

Though the notion of entanglement is an intensively discussed concept of quantum mechanics, it is hard to find in the literature a precise definition of this concept (we refer to [4] for various characterizations of entanglement). Our attempt here is to develop a probabilistic formulation of the entanglement as a specific kind of correlation.

The probabilistic framework we will adopt is summarized in Section 2: it rests on the usual Kolmogorovian formulation of probability theory but it allows a wider family of random variables in order to encompass indeterministic behaviours.

When we speak of correlations we always mean correlations among results of a joint measurement of two or more observables at some state. In the classical

\footnotetext{
${ }^{*}$ Supported by the Polish Committee for Scientific Research (KBN), grant No 7 T11C 017
} 21. 
deterministic case there is no joint observable other than the product joint observable, so that the occurrence of a correlation depends only upon properties that are coded in the state of the physical system. In particular, no correlation can occur when the state is pure, namely when we have to do with a maximal information about the preparation of the physical system. Things are different, as discussed in Section 3, when we deal with indeterministic observables: the choice of a joint observable is, in general, no longer unique and the occurrence of a correlation becomes a function of that choice. New kinds of correlations emerge and the notion of entanglement will appear related to the fact that a non-product joint obseravble can give rise to a correlation even if the physical system is in a pure state.

\section{Probabilistic physical systems}

We provide here a probabilistic framework that appears general eonugh to host the description of physical objects showing an intrinsic random behaviour. As usual, the basic ingredients are the physical notions of states and observables, whose counterparts in the language of probability theory are the probability measures on the sample space and the random variables.

The states of a probabilistic physical object are pragmatically identified with the possible ways of preparing statistical ensembles of samples of the object under discussion. The set of all states carries the natural convex structure which mirrors the possibility of performing statistical mixing of ensembles. We assume that the convex set of states can be identified with (is affinely isomorphic to) the convex set $M_{1}^{+}(\Omega)$ of all probability measures on a measurable space $(\Omega ; \mathcal{B}(\Omega))$, often called the phase space of the physical system $(\mathcal{B}(\Omega)$ stands for a Boolean algebra of measurable subsets of $\Omega$ ). We assume further that every one-point subset of $\Omega$ is measurable, so that $\{\omega\} \in \mathcal{B}(\Omega)$ for every $\omega \in \Omega$. The last assumption implies that all Dirac measures $\delta_{\omega}, \omega \in \Omega$, belong to $M_{1}^{+}(\Omega)$, so representing particular states called pure states. Non-pure states are also called mixed.

Notice that, due to the particular convex structure of $M_{1}^{+}(\Omega)$, a mixed state has the classical property of admitting a unique decomposition into pure states: this alludes to the fact that the family of observable properties of the physical system under discussion will be rich enough to separate the elements of $M_{1}^{+}(\Omega)$. There is no distinguished probability measure on $\Omega$; all probability measures on $\Omega$ are considered as possible states of the probabilistic (randomly behaving) physical system.

We restrict our attention to a special (nevertheless sufficiently large) class of experiments, or measurements with random outcomes (see, for instance, [6], [8]): any measurement on a probabilistic physical system is performed on a state, so on an ensemble of identically prepared samples of the physical system, and results in a probability distribution on the space of the possible outcomes of single individual measurements. Equivalence classes of measurements are called observables (observable properties). Therefore, an abstract description of an 
observable is a map

$$
A: M_{1}^{+}(\Omega) \rightarrow M_{1}^{+}(\Xi)
$$

which transforms states into probability distributions on the space $\Xi$ of the outcomes of the observable; the measurement of $A$ at the state $\mu \in M_{1}^{+}(\Omega)$ will produce the measure $A \mu \in M_{1}^{+}(\Xi)$ to be called the outcome measure. Clearly, the outcome space has to be measurable: we will further assume that every one-point subset of $\Xi$ is measurable, namely, $\{\xi\} \in \mathcal{B}(\Xi)$ for every $\xi \in \Xi$.

All observables we are going to consider react linearly on the mixing of ensembles; thus, an observable should be an affine (i.e. convexity preserving) map, explicitely: $A\left(\lambda \mu_{1}+(1-\lambda) \mu_{2}\right)=\lambda A \mu_{1}+(1-\lambda) A \mu_{2}$ for every $\mu_{1}, \mu_{2} \in$ $M_{1}^{+}(\Omega)$ and every real number $\lambda, 0 \leq \lambda \leq 1$.

It is natural (see [5]) to assume that every observable $A: M_{1}^{+}(\Omega) \rightarrow M_{1}^{+}(\Xi)$ satisfies the condition

$$
(A \mu)(X)=\int_{\Omega}\left(A \delta_{\omega}\right)(X) \mu(d \omega)
$$

for every $X \in \mathcal{B}(\Xi)$ and every state $\mu \in M_{1}^{+}(\Omega)$. This measurability condition expresses the specific property that an observable is uniquely defined by the outcome measures it associates to the pure states.

\subsection{Deterministic and indeterministic observables}

An observable $A: M_{1}^{+}(\Omega) \rightarrow M_{1}^{+}(\Xi)$ will be called deterministic if it assumes a well defined value at every pure state; formally if it maps Dirac measures on $(\Omega ; \mathcal{B}(\Omega))$ into Dirac measures on $(\Xi ; \mathcal{B}(\Xi))$. In other words, the deterministic observables have no dispersion on pure states. The affine character of the observables implies that a deterministic observable can exhibit some randomness, or some dispersion, when it acts on a mixed state. However, this stochastic aspect is fully reducible to the mixed character of the state. It is easy to see that deterministic observables correspond just to $\Xi$-valued random variables of the standard probability theory (see [5]).

Observables which are not deterministic exhibit an inherent randomness: at some pure states they show dispersion, i.e., they generate diffused probability measures on the outcome space.

The distinction between deterministic and indeterministic observables is of a deep nature. The traditional notion of random variable mirrors our notion of deterministic observable: the classical probability theory which admits only deterministic observables describes merely a lack of information in a deterministic world. The concept of non-deterministic observable implies in general a nontrivial statistical dispersion of possible results of an observation even in presence of a maximal information about the preparation of the physical system. The probabilistic framework here introduced, which includes the occurrence of indeterministic observables, belongs to the so called operational probability theory (see $[2],[5])$ and is able to describe an irreducible randomness - either inherent or produced by an uncontrolled outer influence. 
It is evident that typical quantum-mechanical observables have an indeterministic nature. It has been shown elsewhere (see [2]) that the operational probabilistic framework here described can host an extension of quantum mechanics. Notice that if we call $\Omega$ the set of pure states of a quantum system, then the usual family of the quantum-mechanical observables is not rich enough to separate $M_{1}^{+}(\Omega)$ : indeed we know that the usual quantum mixed states have infinitely many convex decompositions into pure states. In the extension of quantum mechanics alluded to, the quantum observables are embedded into the bigger family of the observables on $M_{1}^{+}(\Omega)$, which is actually rich enough to separate distinct convex combinations of pure states.

\subsection{Joint observables}

We have defined an observable without any specific characterization of its outcome space $\Xi$. When the outcome space takes the structure of the Cartesian product $\Xi=\Xi_{1} \times \Xi_{2}$, then the observable $A: M_{1}^{+}(\Omega) \rightarrow M_{1}^{+}\left(\Xi_{1} \times \Xi_{2}\right)$ defines two observables

$$
A_{i}:=\Pi_{i} \circ A, \quad i=1,2,
$$

where $\Pi_{i}: M_{1}^{+}\left(\Xi_{1} \times \Xi_{2}\right) \rightarrow M_{1}^{+}\left(\Xi_{i}\right)$ is the marginal projection, i.e.,

$$
\Pi_{1} \nu\left(X_{1}\right):=\nu\left(X_{1} \times \Xi_{2}\right), \Pi_{2} \nu\left(X_{2}\right):=\nu\left(\Xi_{1} \times X_{2}\right),
$$

for every $\nu \in M_{1}^{+}\left(\Xi_{1} \times \Xi_{2}\right), X_{i} \in \mathcal{B}\left(\Xi_{i}\right), i=1,2$.

The observable $A: M_{1}^{+}(\Omega) \rightarrow M_{1}^{+}\left(\Xi_{1} \times \Xi_{2}\right)$ is called a joint observable of $A_{1}$ and $A_{2}$. It should be stressed, however, that a pair of observables $A_{1}$ : $M_{1}^{+}(\Omega) \rightarrow M_{1}^{+}\left(\Xi_{1}\right), A_{2}: M_{1}^{+}(\Omega) \rightarrow M_{1}^{+}\left(\Xi_{2}\right)$ admits, in general, many joint observables.

A particular joint observable for $A_{1}$ and $A_{2}$ is the product one, denoted $A_{1} \otimes A_{2}$, and defined by

$$
A_{1} \otimes A_{2} \delta_{\omega}:=A_{1} \delta_{\omega} \otimes A_{2} \delta_{\omega}
$$

for every $\omega \in \Omega$ (see [2],[5]), the symbol $\otimes$ in the r.h.s. standing for the usual product of measures (if $\nu_{i} \in M_{1}^{+}\left(\Xi_{i}\right), i=1,2$, then $\nu_{1} \otimes \nu_{2}\left(X_{1} \times X_{2}\right):=$ $\nu_{1}\left(X_{1}\right) \cdot \nu_{2}\left(X_{2}\right)$ for every $\left.X_{i} \in \mathcal{B}\left(\Xi_{i}\right), i=1,2\right)$. The definition is exhaustive thanks to the condition of measurability expressed by Eq.(1). As the product observable $A_{1} \otimes A_{2}$ does exist for every pair $A_{1}, A_{2}$, any two observables pertaining to a probabilistic physical system are comeasurable, what is commonly considered as a feature characterizing "classical" theories. In this sense the present framework behaves as "classical" though only the deterministic observables have a counterpart in classical mechanics and in standard classical probability theory.

Any pair of deterministic observables has a unique joint observable, the product observable, in full agreement with the standard probability theory (where the joint observable is usually called the "random vector"), while a joint observable of two indeterministic observables is non-unique. 
Writing $J\left(A_{1}, A_{2}\right)$ to denote a joint observable of $A_{1}: M_{1}^{+}(\Omega) \rightarrow M_{1}^{+}\left(\Xi_{1}\right)$ and $A_{2}: M_{1}^{+}(\Omega) \rightarrow M_{1}^{+}\left(\Xi_{2}\right)$, the measure $J\left(A_{1}, A_{2}\right) \mu \in M_{1}^{+}\left(\Xi_{1} \times \Xi_{2}\right)$, i.e. the statistical result of the measurement of $J\left(A_{1}, A_{2}\right)$ at $\mu \in M_{1}^{+}(\Omega)$, clearly determines the statistical results of measurements of both $A_{1}$ and $A_{2}$ at the state $\mu$. Thus, the joint observable $J\left(A_{1}, A_{2}\right)$ can be interpreted as a particular method for a simultaneous measurement of $A_{1}$ and $A_{2}$. Various joint observables would then correspond to various ways of performing simultaneous measurements.

On the other hand, the fact that $A_{1} \mu$ and $A_{2} \mu$ do not determine uniquely $J\left(A_{1}, A_{2}\right) \mu$ suggests that a simultaneous measurement of $A_{1}$ and $A_{2}$ could even be performed directly, without measuring any joint observable of them. Indeed, one could measure $A_{1}$ on every second member of the statistical ensemble $\mu$, and $A_{2}$ on the other members. Such a measurement provides directly two probability measures $A_{1} \mu$ and $A_{2} \mu$ without any joint measure of them.

This means that the concept of a simultaneous measurement of two or more observables does not coincide with the concept of a joint measurement. The former is surely more general than the latter, but a measuement of a joint observable is more "informative": it provides also the correlations that might emerge between, or among, the observables.

\section{Probabilistic correlations}

We assume the common-sense concept of correlation: two parameters are correlated whenever they are not independent. The notion of correlation can be understood in various ways, for instance in algebraic terms as a (non-trivial) relation, or as a fuzzy relation, but here we mean the probabilistic version of that concept; hence we speak about probabilistic (or stochastic) correlation and probabilistic (or stochastic) independence.

Consider two parameters and let $\Xi_{1}, \Xi_{2}$ be the (measurable) sets of their possible values; given a measure $\nu \in M_{1}^{+}\left(\Xi_{1} \times \Xi_{2}\right)$ we say, according to the standard probability theory, that the two parameters are mutually independent relative to $\nu$ iff

$$
\nu=\Pi_{1} \nu \otimes \Pi_{2} \nu,
$$

explicitely, iff $\nu\left(X_{1} \times X_{2}\right)=\left(\Pi_{1} \nu\right)\left(X_{1}\right) \cdot\left(\Pi_{2} \nu\right)\left(X_{2}\right)$ for every $X_{i} \in M_{1}^{+}\left(\Xi_{i}\right), i=$ 1,2 . If $\nu$ has not this product form, the two parameters will be considered correlated relative to $\nu$.

It is an immediate consequence of this definition the fact that the two parameters are independent at every Dirac measure $\delta_{\left(\xi_{1}, \xi_{2}\right)}$ because

$$
\delta_{\left(\xi_{1}, \xi_{2}\right)}=\delta_{\xi_{1}} \otimes \delta_{\xi_{2}}, \text { for every } \xi_{1} \in \Xi_{1}, \xi_{2} \in \Xi_{2} .
$$

Thus we see that two parameters can be correlated only relative to a measure $\nu \in M_{1}^{+}\left(\Xi_{1} \times \Xi_{2}\right)$ having the form of a mixture; notice however that the mixed

nature of $\nu$ is not a sufficient condition to produce correlation (for instance, if $\Xi_{1}=\Xi_{2}$ we have independence relative to the symmetrized mixture $\frac{1}{4}\left(\delta_{\left(\xi_{1}, \xi_{1}\right)}+\right.$ $\left.\left.\delta_{\left(\xi_{1}, \xi_{2}\right)}+\delta_{\left(\xi_{2}, \xi_{1}\right)}+\delta_{\left(\xi_{2}, \xi_{2}\right)}\right)\right)$. 
Let us stress that our definition of correlation as the negation of independence does not overlap exactly with the traditional one assumed in standard probability theory, where a correlation coefficient is introduced which quantifies to what extent the joint distribution $\nu \in M_{1}^{+}\left(\Xi_{1} \times \Xi_{2}\right)$ deviates from a one concentrated on a line, so that $\nu$ is said to carry a correlation if such a coefficient does not vanish. If $\nu=\Pi_{1} \nu \otimes \Pi_{2} \nu$, namely if the parameters are independent relative to $\nu$, then the correlation coefficient equals zero. This always happens if $\nu$ is a Dirac measure. However, the vanishing of the correlation coefficient does not imply that $\nu=\Pi_{1} \nu \otimes \Pi_{2} \nu$; simple examples can be found in textbooks (see, e.g., [9], pp. 328 - 9). That means that the correlation coefficient provides only a coarse characterization of the concept of correlation adopted here.

Let us now come to the notion of independence and correlation of observables. A pair of observables, say $A_{1}: M_{1}^{+}(\Omega) \rightarrow M_{1}^{+}\left(\Xi_{1}\right), A_{2}: M_{1}^{+}(\Omega) \rightarrow$ $M_{1}^{+}\left(\Xi_{2}\right)$, does not produce at a state $\mu \in M_{1}^{+}(\Omega)$ any well defined measure on the product of the outcome sets $\Xi_{1} \times \Xi_{2}$. Consequently, it makes no sense to speak of independence (or correlation) of two observables without reference to some joint observable: the concept of independence should be relative to a particular joint observable, so to a particular way the two observables are paired together. Thus, the notion of independence expressed by Eq.(3) brings us to the following definition:

Two observables $A_{1}: M_{1}^{+}(\Omega) \rightarrow M_{1}^{+}\left(\Xi_{1}\right), A_{2}: M_{1}^{+}(\Omega) \rightarrow M_{1}^{+}\left(\Xi_{2}\right)$ are independent at the state $\mu \in M_{1}^{+}(\Omega)$ relative to the joint observable $J\left(A_{1}, A_{2}\right)$ : $M_{1}^{+}(\Omega) \rightarrow M_{1}^{+}\left(\Xi_{1} \times \Xi_{2}\right)$ iff $J\left(A_{1}, A_{2}\right) \mu=A_{1} \mu \otimes A_{2} \mu$.

Accordingly, we say that two observables $A_{1}$ and $A_{2}$ are correlated (mutually dependent) at the state $\mu$ relative to the joint observable $J\left(A_{1}, A_{2}\right)$ iff

$$
J\left(A_{1}, A_{2}\right) \mu \neq A_{1} \mu \otimes A_{2} \mu .
$$

Though familiar examples of correlated observables often refer to compound systems, it should be noticed that the above concept of correlation does not require any restriction about the nature of the physical system.

\subsection{Classical correlations and probabilistic entanglement}

Among the possible joint observables of $A_{1}$ and $A_{2}$ there is always the product $A_{1} \otimes A_{2}$ characterized by the property expressed by Eq.(2) which ensures the independence of any two observables $A_{1}, A_{2}$ at every pure state, relative to $A_{1} \otimes A_{2}$. Clearly, for a nonpure state $\mu \in M_{1}^{+}(\Omega)$, the measure $A_{1} \otimes A_{2} \mu$ has the form of a mixture and, as already noticed, we have, in general,

$$
A_{1} \otimes A_{2} \mu \neq A_{1} \mu \otimes A_{2} \mu .
$$

In view of the independence of $A_{1}$ and $A_{2}$ at every pure state, the occurrence of a correlation in $A_{1} \otimes A_{2} \mu$ has to be interpreted as due to properties coded in the mixed state $\mu$. Thus, we can view the joint observable $A_{1} \otimes A_{2}$ as representing the correlation-free way of pairing $A_{1}$ and $A_{2}$, because a correlation appearing 
in $A_{1} \otimes A_{2} \mu$ is generated by the state $\mu$, namely by the way the pure states are mixed together to form $\mu$.

In the standard classical case, where only deterministic observables come into play, the product $A_{1} \otimes A_{2}$ is known to be the unique joint observable of $A_{1}, A_{2}$ : the correlation carried by $A_{1} \otimes A_{2} \mu$ thus becomes the only possible correlation. This is why this correlation, which depends only on properties coded in the mixed state $\mu$ of the physical system, will be called the classical correlation.

Clearly, when we speak of the classical correlation carried by $A_{1} \otimes A_{2} \mu$ we are not committed to assume that $A_{1}$ and $A_{2}$ are deterministic: the classical correlation can appear also in a nonderministic framework.

But when we deal with two indeterministic observables $A_{1}, A_{2}$, the product $A_{1} \otimes A_{2}$ is no longer the only possible joint observable. A joint observable $J\left(A_{1}, A_{2}\right)$ differing from $A_{1} \otimes A_{2}$ can produce at a state $\mu$ a probability distribution $J\left(A_{1}, A_{2}\right) \mu$ carrying an additional correlation, which will depend on the particular way the two observables are paired together to form $J\left(A_{1}, A_{2}\right)$. Thus, we expect that the correlation contained in the outcome measure $J\left(A_{1}, A_{2}\right) \mu$ can be described as coming from two mutually independent factors: (i) the classical correlation inherent to the state $\mu$, and (ii) the possible additional correlation, to be called probabilistic entanglement, introduced by the joint observable in question. Due to this second factor the total correlation contained in the distribution $J\left(A_{1}, A_{2}\right) \mu$ should be different for different joint observables of $A_{1}$ and $A_{2}$.

Typical of the probabilistic entanglement, associated to a joint observable differing from $A_{1} \otimes A_{2}$, is the fact that it need not vanish at pure states. Since the classical correlation vanishes at the pure states, any correlation appearing at a pure state has to be recognized as a probabilistic entanglement.

The occurrence of correlations at pure states is a characteristic feature of the quantum behaviour: the "entangled states" of the quantum dictionary are indeed pure states (of a compound system) and correlations can appear at these states. Let us stress that familiar expressions like "a state shows entanglement" make sense only if a joint observable is implicitly referred to (compare with [7]) and, similarly, one could say that "a joint observable shows entanglement" only if a state is implicitly referred to.

Notice that the familiar EPR, or Bell, correlation is indeed a correlation occurring at a pure state of a compound system (an "entangled" state) and relative to a joint observable (the tensor product of the two observables involved) which is not a product joint observable in the sense of Eq.(2). What we have called probabilistic entanglement encompasses the quantum idea of entanglement, casting it in a more general framework.

Summing up, given two observables $A_{1}: M_{1}^{+}(\Omega) \rightarrow M_{1}^{+}\left(\Xi_{1}\right), A_{2}: M_{1}^{+}(\Omega) \rightarrow$ $M_{1}^{+}\left(\Xi_{2}\right)$, given a joint observable $J\left(A_{1}, A_{2}\right) \neq A_{1} \otimes A_{2}$, and given a state $\mu \in M_{1}^{+}(\Omega)$, we focus attention on three probability measures on $\Xi_{1} \times \Xi_{2}$ :

$$
A_{1} \mu \otimes A_{2} \mu, \quad A_{1} \otimes A_{2} \mu, \quad J\left(A_{1}, A_{2}\right) \mu .
$$

The measure $A_{1} \mu \otimes A_{2} \mu$ can be seen as the result of performing an indepen- 
dent measurement of $A_{1}$ and $A_{2}$ at $\mu$ and taking formally the product of the two obtained measures (a joint observable giving the result $A_{1} \mu \otimes A_{2} \mu$ when measured at $\mu$ need not exist). On the contrary, the measures $A_{1} \otimes A_{2} \mu$ and $J\left(A_{1}, A_{2}\right) \mu$ can be naturally seen as the result of measuring the product joint observable $A_{1} \otimes A_{2}$ and, respectively, a non-product joint observable $J\left(A_{1}, A_{2}\right)$ at $\mu$. Obviously, all three measures give back $A_{1} \mu$ and $A_{2} \mu$ as marginals.

What we have called classical correlation mirrors the departure betwen the two measures $A_{1} \otimes A_{2} \mu$ and $A_{1} \mu \otimes A_{2} \mu$; what we have called probabilistic entanglement mirrors the departure between the two measures $J\left(A_{1}, A_{2}\right) \mu$ and $A_{1} \otimes A_{2} \mu$. The total correlation at a state $\mu$ relative to the (non-product) joint observable $J\left(A_{1}, A_{2}\right)$ mirrors the departure between $J\left(A_{1}, A_{2}\right) \mu$ and $A_{1} \mu \otimes A_{2} \mu$. In the next Section the separation between classical correlation and probabilistic entanglement will be made clear by introducing their density functions.

\subsection{Correlation density functions}

We will be interested in comparing two measures, say $\tilde{\nu}, \nu$, on a measurable space having the form $\Xi_{1} \times \Xi_{2}$. The Radon-Nikodym, or density function, of $\tilde{\nu}$ relative to $\nu$ is a function $d \tilde{\nu} / d \nu: \Xi_{1} \times \Xi_{2} \rightarrow \mathbf{R}^{+}$such that

$$
\tilde{\nu}(X)=\int_{X} \frac{d \tilde{\nu}}{d \nu} \cdot \nu\left(d\left(\xi_{1}, \xi_{2}\right)\right) \text { for every } X \in \mathcal{B}\left(\Xi_{1} \times \Xi_{2}\right) .
$$

This Radon-Nikodym derivative provides a complete description of the measure $\tilde{\nu}$ (given $\nu$ ), hence it also contains all informations on the correlations inherent to $\tilde{\nu}$.

Let us now come to the three measures $A_{1} \mu \otimes A_{2} \mu, A_{1} \otimes A_{2} \mu$, and $J\left(A_{1}, A_{2}\right) \mu$ discussed in the previous section, and consider the three density functions from $\Xi_{1} \times \Xi_{2}$ into $\mathbf{R}^{+}$(provided they exist, see the Appendix):

$$
\rho_{c}:=\frac{d\left(A_{1} \otimes A_{2} \mu\right)}{d\left(A_{1} \mu \otimes A_{2} \mu\right)}, \quad \rho_{e}:=\frac{d\left(J\left(A_{1}, A_{2}\right) \mu\right)}{d\left(A_{1} \otimes A_{2} \mu\right)}, \quad \rho_{t}:=\frac{d\left(J\left(A_{1}, A_{2}\right) \mu\right)}{d\left(A_{1} \mu \otimes A_{2} \mu\right)}
$$

The classical correlation is fully characterized by the density function $\rho_{c}$, which describes the departure between the measure $A_{1} \otimes A_{2} \mu$ associated to the product joint observable and the measure $A_{1} \mu \otimes A_{2} \mu$ in which $A_{1}, A_{2}$ behave as independent. Thus, we call $\rho_{c}$ the classical-correlation function.

The departure between the measures $J\left(A_{1}, A_{2}\right) \mu$ and $A_{1} \otimes A_{2} \mu$ is fully described by the density function $\rho_{e}$ which thus characterizes the probabilistic entanglement of the observables $A_{1}, A_{2}$ at the state $\mu$ relative to the (nonproduct) joint observable $J\left(A_{1}, A_{2}\right)$. The density function $\rho_{e}$ will thus be called the entanglement function.

As already remarked, the total correlation inherent to $J\left(A_{1}, A_{2}\right)$ at $\mu$ emerges by comparing the measure $J\left(A_{1}, A_{2}\right) \mu$ with the measure $A_{1} \mu \otimes A_{2} \mu$ which carries no correlation. An exhaustive description of this correlation is then provided by the density function $\rho_{t}$ which we will call the total-correlation function.

On the other hand, we have seen that the correlations shown by $J\left(A_{1}, A_{2}\right) \mu$ can be divided into two parts, characterized by the two functions $\rho_{c}$ and $\rho_{e}$ 
introduced above. The three correlation functions are connected together: indeed, the general theory of the Radon-Nikodym derivatives states that (see $e, g$. [1], Corollary 2.9.4, or [3], Sect. 32)

$$
\rho_{t}=\rho_{c} \cdot \rho_{e} .
$$

This simple product formula provides an a posteriori support for the ideas expressed in the previous Section.

Notice that for a pure state $\mu=\delta_{\omega}$ we have: $A_{1} \otimes A_{2} \delta_{\omega}=A_{1} \delta_{\omega} \otimes A_{2} \delta_{\omega}$, so that the classical-correlation function $\rho_{c}$ becomes the constant unit function and we get $\rho_{t}=\rho_{e}$.

The calculation of the correlation functions might be hard for a general case, but concrete situations of interest often involve finite sets of values as well as pure states or finite mixtures, what makes the calculations handable.

Let the two sets $\Xi_{1}, \Xi_{2}$ be finite and let $\xi_{1} \in \Xi_{1}, \xi_{2} \in \Xi_{2}$ : if, for instance, we are concerned with the entanglement function relative to the joint observable $J\left(A_{1}, A_{2}\right): M_{1}^{+}(\Omega) \rightarrow M_{1}^{+}\left(\Xi_{1} \times \Xi_{2}\right)$, then we can write

$$
\left(J\left(A_{1}, A_{2}\right) \mu\right)(X)=\sum_{\left(\xi_{1}, \xi_{2}\right) \in X} \rho_{e} \cdot\left(A_{1} \otimes A_{2} \mu\right)\left(\xi_{1}, \xi_{2}\right)
$$

for every $X \in \Xi_{1} \times \Xi_{2}$. This makes clear that the entanglement function $\rho_{e}$ will take the form

$$
\rho_{e}\left(\xi_{1}, \xi_{2}\right)=\frac{\left(J\left(A_{1}, A_{2}\right) \mu\right)\left(\xi_{1}, \xi_{2}\right)}{\left(A_{1} \otimes A_{2} \mu\right)\left(\xi_{1}, \xi_{2}\right)} .
$$

If the state $\mu$ is a finite mixture of pure states, say $\mu=\sum_{i} \lambda_{i} \delta_{\omega_{i}}$, then the above numerator and denominator will take the corresponding form of finite sums, since $J\left(A_{1}, A_{2}\right)$ and $A_{1} \otimes A_{2}$ act affinely on $M_{1}^{+}(\Omega)$.

Let us finally remark that the density functions introduced before can be used to produce various numerical characterizations of the corresponding correlations.

\section{Appendix}

We collect here some technical results on the existence of the Radon-Nikodym derivatives used in Section 3.

LEMMA: If $\nu \in M_{1}^{+}\left(\Xi_{1} \times \Xi_{2}\right)$ is a discrete measure and $\nu_{1}:=\Pi_{1} \nu, \nu_{2}:=\Pi_{2} \nu$ are its marginals, then $\nu \ll \nu_{1} \otimes \nu_{2}$ (i.e., $\nu$ is absolutely continuous w.r.t. $\nu_{1} \otimes \nu_{2}$ ).

Proof Recall that the defining property of the product measure $\nu_{1} \otimes \nu_{2}$ is:

$$
\left(\nu_{1} \otimes \nu_{2}\right)\left(X_{1} \times X_{2}\right)=\nu_{1}\left(X_{1}\right) \nu_{2}\left(X_{2}\right)
$$

for every $X_{1} \in \mathcal{B}\left(\Xi_{1}\right), X_{2} \in \mathcal{B}\left(\Xi_{2}\right)$. If $\nu\left(\xi_{1}, \xi_{2}\right)$ and $\nu\left(\eta_{1}, \eta_{2}\right)$ are both non zero $\left(\xi_{1}, \eta_{1} \in \Xi_{1}, \xi_{2}, \eta_{2} \in \Xi_{2}\right)$, then $\nu_{1}\left(\xi_{1}\right), \nu_{1}\left(\eta_{1}\right), \nu_{2}\left(\xi_{2}\right), \xi_{2}\left(\eta_{2}\right)$ are all non zero so that $\left(\nu_{1} \otimes \nu_{2}\right)$ is different from zero at all points of the set $\left\{\xi_{1}, \xi_{2}\right\} \times\left\{\eta_{1}, \eta_{2}\right\}$. This implies that $\nu(X)=0$ for some $X \in \mathcal{B}\left(\Xi_{1} \times \Xi_{2}\right)$ implies $\left(\nu_{1} \otimes \nu_{2}\right)(X)=0$, what is equivalent to $\nu \ll \nu_{1} \otimes \nu_{2}$. 
Notice that the discreteness restriction is crucial as the following counterexample (suggested by P.J. Lahti and A. Dvurecenskij) shows. Take for $\Xi_{1}$ and $\Xi_{2}$ the real interval $[0,1]$, let $\nu_{1}, \nu_{2}$ be Lebesgue measures on $\Xi_{1}, \Xi_{2}$ respectively, and define $\nu \in M_{1}^{+}\left(\Xi_{1} \times \Xi_{2}\right)$ by $\nu\left(X_{1} \times X_{2}\right):=\nu_{1}\left(X_{1} \cap X_{2}\right)$ (where $X_{2} \subseteq \Xi_{2}$ is identified with the corresponding subset of $\left.\Xi_{1}\right)$ so that $\nu$ is concentrated at the main diagonal $\{(\xi, \xi) \mid \xi \in[0,1]\}$ of the square $\Xi_{1} \times \Xi_{2}$. Clearly, $\nu_{1}$ and $\nu_{2}$ are marginals of $\nu$, however $\nu_{1} \otimes \nu_{2}$ is the Lebesgue measure on $\Xi_{1} \times \Xi_{2}$ that vanishes on the main diagonal so that $\nu$ is not absolutely continuous w.r.t. $\nu_{1} \otimes \nu_{2}$.

The property stated in this Lemma ensures the existence of the RadonNikodym derivative $d \nu / d\left(\nu_{1} \otimes \nu_{2}\right)$ which is (see [1], Theorem 2.5.5) $\nu_{1} \otimes \nu_{2^{-}}$ almost everywhere finite. This result can be applied to ensure the existence of the density functions $\rho_{c}$ and $\rho_{t}$ occurring in Eqs.(6), provided the measures $A_{1} \otimes A_{2} \mu$ and $J\left(A_{1}, A_{2}\right) \mu$ are discrete.

Concerning the entanglement function $\rho_{e}$ we make use of the following

COROLLARY: Let $J\left(A_{1}, A_{2}\right): M_{1}^{+}(\Omega) \rightarrow M_{1}^{+}\left(\Xi_{1} \times \Xi_{2}\right)$ be a discrete joint observable of $A_{1}$ and $A_{2}$. Then, for every $\mu \in M_{1}^{+}(\Omega)$, we have $J\left(A_{1}, A_{2}\right) \mu \ll$ $A_{1} \otimes A_{2} \mu$

Proof In view of Eq.(1)) we have that $\left(A_{1} \otimes A_{2} \mu\right)(X)=0$ implies $\left(A_{1} \otimes\right.$ $\left.A_{2} \delta_{\omega}\right)(X)=0, \mu$-almost everywhere. Since the previous Lemma ensures that

$$
J\left(A_{1}, A_{2}\right) \delta_{\omega} \ll A_{1} \delta_{\omega} \otimes A_{2} \delta_{\omega}=A_{1} \otimes A_{2} \delta_{\omega}
$$

we also have $\left(J\left(A_{1}, A_{2}\right) \delta_{\omega}\right)(X)=0, \mu$-almost everywhere. Looking again at Eq.(1) we conclude that $\left(A_{1} \otimes A_{2} \mu\right)(X)=0$ implies $\left(J\left(A_{1}, A_{2}\right) \mu\right)(X)=0$ for every measurable subset $X \in \mathcal{B}\left(\Xi_{1} \times \Xi_{2}\right)$.

This corollary ensures the existence of the entanglement function $\rho_{e}$ occurring in Eq.(6) for every discrete joint observable $J\left(A_{1}, A_{2}\right)$.

\section{References}

[1] H. Bauer, Probability Theory and Elements of Measure Theory, Academic Press, London, 1981.

[2] E. G. Beltrametti, S. Bugajski, J. Phys. A: Math.Gen. 28 (1995) 3329 3343. S. Bugajski, Int. J. Theor. Phys. 35 (1996) 2229 - 2244.

[3] P. Billingsley, Probability and Measure, J.Wiley, New York, 1979.

[4] D. Bruß: Characterizing entanglement, arXiv:quant-ph/0110078 v1 12 Oct 2001.

[5] S. Bugajski, Mathematica Slovaca 51 (2001) 321 - 342 and 343 - 361.

[6] P. Busch, P. J. Lahti, P. Mittelstaedt, The Quantum Theory of Measurement, Lecture Notes in Physics, 2nd Edition, Springer-Verlag, Berlin, 1996.

[7] A. Cabello, Phys. Rev. A 60 (1999) 877 - 880.

[8] A. S. Holevo, Probabilistic and Statistical Aspects of Quantum Theory, Statistics and Probability 1, North-Holland, Amsterdam, 1982.

[9] P. E. Pfeiffer, Concepts of Probability Theory, McGraw-Hill, New York, 1965. 